\title{
Synthesis and Properties of Magnetic Carbon Nanocages Particles for Dye Removal
}

\author{
Hengfei Qin, ${ }^{1}$ Yongkui Huang, ${ }^{1}$ Siyu Liu, ${ }^{1}$ Yao Fang, ${ }^{1}$ Xi Li, ${ }^{1}$ and Shifei Kang ${ }^{2}$ \\ ${ }^{1}$ Department of Environmental Science and Engineering, Fudan University, Shanghai 200433, China \\ ${ }^{2}$ Department of Environmental Science and Engineering, University of Shanghai for Science and Technology, \\ Shanghai 200093, China
}

Correspondence should be addressed to Xi Li; xi_i@fudan.edu.cn and Shifei Kang; karmatimes@126.com

Received 30 May 2015; Revised 21 July 2015; Accepted 29 July 2015

Academic Editor: Yuan Chen

Copyright (C) 2015 Hengfei Qin et al. This is an open access article distributed under the Creative Commons Attribution License, which permits unrestricted use, distribution, and reproduction in any medium, provided the original work is properly cited.

\begin{abstract}
Magnetic carbon nanocages (MCNCs) with multiform pore structure have been synthesized by a simple low temperature carbonization process. Biorenewable lignin was used as a cheap and carbon-rich precursor for the first time. The products were characterized by X-ray diffraction (XRD), nitrogen adsorption-desorption, energy dispersive X-ray spectroscopy (EDS), vibrating sample magnetometer (VSM), transmission electron microscopy (TEM), and Raman spectrum. XRD pattern and Raman spectrum showed that the product has a high degree of graphitization crystallinity. TEM micrograph indicated that the synthesized MCNCs have the hierarchical pore and cage structure. Due to these characteristics, the obtained magnetic carbon nanocages can be used as efficient and recycled adsorbents in the removal of dye staff from textile wastewater.
\end{abstract}

\section{Introduction}

Carbon materials, in their various forms such as carbon nanocages [1], porous materials [2], graphene [3], nanotubes [4], fibers [5], and activated carbons [6], had good adsorption, low density, high surface area, excellent electrical conductivity, and efficient catalytic activity. Among them are carbon nanocages (CNCs), an emerging engineered material, generally used as catalyst supports [7-11], adsorbents [12], electrode materials [13], lithium-ion batteries [14-19], supercapacitors [20,21], drug delivery [22, 23], and sensors [24].

To date, various synthesis methods, for instance, chemical vapor deposition (CVD) [25], arc discharge, template approach [26], pyrolysis of organic precursors [27], and solgel method [28], had been developed to fabricate carbon nanocages. However, in most of these synthesis approaches, acetylene, ethanol, and pyridine were used as carbon sources, which are expensive and unsustainable fossil fuels; they may not be sufficient in near future. Furthermore, energy and environmental concerns have initiated and energized the research on the development of CNCs from renewable carbon sources. As a result, it is crucial to switch over to the cheap and biorenewable carbon source.

Lignin, a cheap, nontoxic, and carbon-rich renewable resource, is the second most abundant biopolymer after cellulose in the nature [29]. Lignin is usually a waste discharged from paper and biomass bioethanol factories. However, the molecular structure of lignin consists of repeating units of the p-hydroxyphenyl $(\mathrm{H})$, guaiacyl $(\mathrm{G})$, and syringyl (S) (Figure 1) [30], which had a highly cross-linked structure close to the network of phenol resin [31, 32]. Thus, the reaction activity of lignin is similar to phenol. Phenol is a kind of toxic chemical intermediates, which was used to synthesize carbon materials [33-35]. Clearly, nontoxic and carbon-rich renewable lignin could replace the phenol as the precursor of carbon materials. Therefore, there are numerous reports of the use of lignin for the preparation of carbon fibers [36], activated carbons [37], carbon nanotube [38], and porous carbon materials [39]. Nevertheless, to the best of our knowledge, the carbon nanocages prepared by lignin have not yet been reported.

Herein, in this paper, for the first time we report a simple, low-cost, and environment-friendly method for preparing 
<smiles>COc1cc([N+](=O)[O-])ccc1O</smiles>

FIGURE 1: Lignin monomeric units.

magnetic carbon nanocages (MCNCs) by a low temperature carbonization process using lignin as the precursor. The structural properties of the obtained samples were characterized by nitrogen adsorption-desorption, X-ray diffraction, transmission electron microscopy, and Raman spectroscopy. Furthermore, lignin-based multiform pore structure and magnetic carbon nanocages used as the adsorbents of methyl orange were investigated.

\section{Experimental}

2.1. Synthesis of Magnetic Carbon Nanocages. All the chemicals were used as received without further purification. In a typical synthesis process: $1.0 \mathrm{~g}$ of lignin was added to $0.35 \mathrm{~g}$ of $20 \mathrm{wt} \% \mathrm{NaOH}$ solution under stirring at $50^{\circ} \mathrm{C}$, after $10 \mathrm{~min}, 1.15 \mathrm{~g}$ of formalin solution ( $37 \mathrm{wt} \%$ formalin) was added dropwise, and the reaction mixture was stirred at $70^{\circ} \mathrm{C}$ for $60 \mathrm{~min}$. Upon cooling the mixture to $30^{\circ} \mathrm{C}, 0.21 \mathrm{~g}$ $\mathrm{Fe}\left(\mathrm{NO}_{3}\right)_{2} \cdot 9 \mathrm{H}_{2} \mathrm{O}$ was added to the mixture. After stirring for $30 \mathrm{~min}$, the dark-brown oligomer was further polymerized at $100^{\circ} \mathrm{C}$ for $12 \mathrm{~h}$ and then peeled off and pyrolyzed at $600^{\circ} \mathrm{C}$ for $3 \mathrm{~h}$ with a ramping rate of $1^{\circ} \mathrm{C} / \mathrm{min}$ under argon flow. Finally, the obtained material was washed with excess amounts of $1 \mathrm{M} \mathrm{HNO}_{3}$ aqueous solution, deionized water and ethanol until a neutral $\mathrm{pH}$, and dried in air at $100^{\circ} \mathrm{C}$ for $12 \mathrm{~h}$. The produced sample was denoted as MCNCs. Commercial activated carbon (AC, Hebei Hua Jing activated Carbon Co., $\left.\mathrm{Ltd}, \mathrm{S}_{\mathrm{BET}}: 800 \mathrm{~m}^{2} \cdot \mathrm{g}^{-1}\right)$ was used as the reference material.

2.2. Measurements and Characterizations. The structural properties of the obtained MCNCs materials were identified using a Rigaku D/Max2rB-II X-ray diffractometer (XRD, CuK1 radiation, $\lambda=1.5406 \AA$ ), operated at $40 \mathrm{kV}$ and $100 \mathrm{~mA}$ (scanning step $8^{\circ} / \mathrm{s}$ ). Transmission electron microscope (TEM) images were recorded using a JEOL JEM-2010 electron microscope with an acceleration voltage of $200 \mathrm{kV}$. The Raman spectrum was recorded on a Horiba XploRA Raman microscope using a $532 \mathrm{~nm}$ argon ion laser. Nitrogen sorption isotherms were measured at $-196^{\circ} \mathrm{C}$ on a Micromeritics ASAP 2000 apparatuses. The Brunauer-Emmett-Teller (BET) method was utilized to calculate the specific surface areas. The pore size distributions were derived from the desorption branches of the isotherms using the Barrett-Joyner-Halenda (BJH) method. VSM measurements were performed by using a vibrating sample magnetometer (Lake Shore 7410 VSM), and the magnetic property of nanocomposite is measured by a strong magnet. The absorbance of methyl orange was measured by 752 type spectrophotometer whose operating voltage was AC $220 \mathrm{~V} / 50 \mathrm{~Hz}$.

2.3. Adsorption of Methyl Orange. In order to investigate the effect of important parameters (e.g., contact time and initial dye concentration) for the removal of MO dye, batch experiments were conducted. For each experimental run, the adsorbent $(50 \mathrm{mg})$ and $\mathrm{MO}(50 \mathrm{~mL})$ stock solutions were taken in a $250 \mathrm{~mL}$ stopper conical flask. This mixture was stirred at a constant speed for 4 hours at room temperature and then the mixture was separated by magnetic separation and the obtained supernatants were tested using a spectrophotometer at wavelength $461.0 \mathrm{~nm}$. Particularly, the representative adsorbent (MCNCs) was withdrawn at different time intervals (0-200 $\mathrm{min})$, separated, and analyzed for remaining dye concentration.

According to the decolorization rate formula for calculation and analysis, the corresponding curve was drawn and then the saturated adsorption capacity was calculated. The removal percentage, defined as the ratio of difference in initial $\mathrm{MO}$ concentration before and after adsorption $\left(C_{0}-C\right)$ to the initial $\mathrm{MO}$ concentration in the aqueous solution $\left(C_{0}\right)$, was calculated using the following equation, while $A_{0}$ and $A$, respectively, represent the absorbance of $\mathrm{MO}$ before and after the adsorption $[40,41]$ :

$$
Q=\left(1-\frac{C}{C_{0}}\right) \times 100 \%=\left(1-\frac{A}{A_{0}}\right) \times 100 \%
$$

Besides, the equilibrium adsorption capacities $\left(Q_{e}\right)$ were determined according to the following formula [42]:

$$
Q_{e}=\frac{\left(C_{i}-C_{e}\right) V}{M}
$$

wherein $C_{i}$ is the initial concentration, $C_{e}$ is the residual concentration, $V$ is the volume of the solution, and $M$ is the mass of the adsorbent.

In research on magnetic separability, $25 \mathrm{mg}$ of adsorbent was added to $25 \mathrm{~mL}$ of $\mathrm{MO}$ dye solution $(50 \mathrm{mg} / \mathrm{L})$ in a bottle. The resulting mixture was stirred at room temperature for $60 \mathrm{~min}$ to reach adsorption equilibrium. Subsequently, a conventional laboratory magnet was placed near the glass bottle, and the solid-liquid separation was achieved within few minutes [41].

\section{Results and Discussion}

The magnetic carbon nanocages (MCNCs) with graphitic framework were evidenced by TEM analysis as shown in Figure 2. As can be seen, the sample consists of nanocages. The size and shape of the nanocages were not uniform, and the size of the nanocages was estimated to be $30-70 \mathrm{~nm}$ (Figure 2(a)). After acid treatment, a great deal of iron phase was eliminated, leaving a graphitic carbon nanocages structure as shown in Figure 2(b), but a small part of iron still residue in the graphitic carbon nanocages. Particularly, the $\mathrm{Fe}_{3} \mathrm{C}$ particles remain within the cages as it is not in an ionic form after the acid treatment of $\mathrm{Fe}_{3} \mathrm{C}$ by $\mathrm{HNO}_{3}$. 


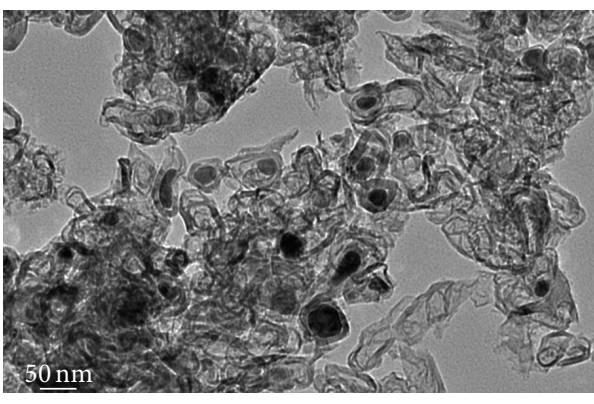

(a)

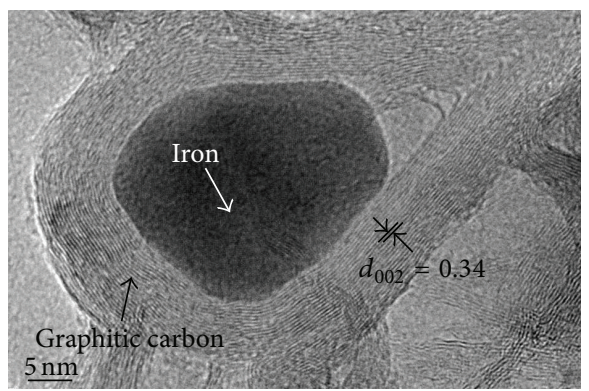

(c)

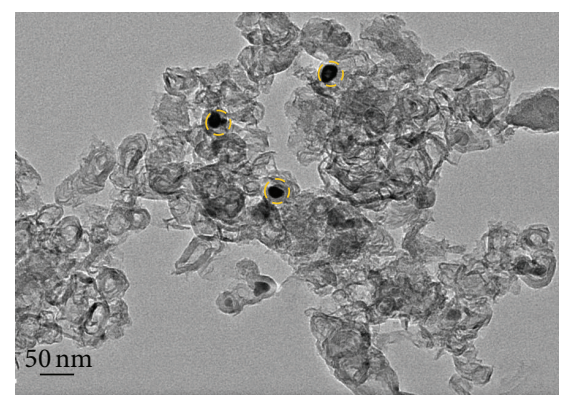

(b)

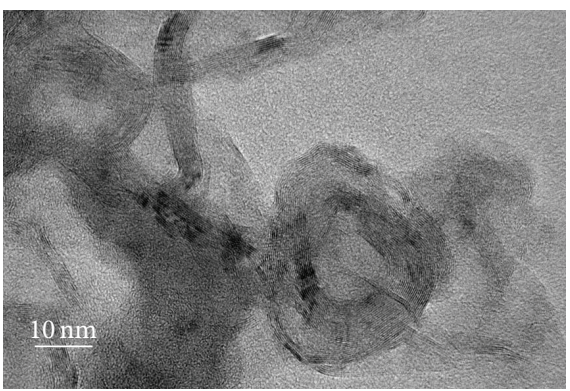

(d)

FIGURE 2: (a) TEM and (c) HRTEM image of magnetic carbon nanocages before acid treatment; (b) TEM and (d) HRTEM image of magnetic carbon nanocages after acid treatment.

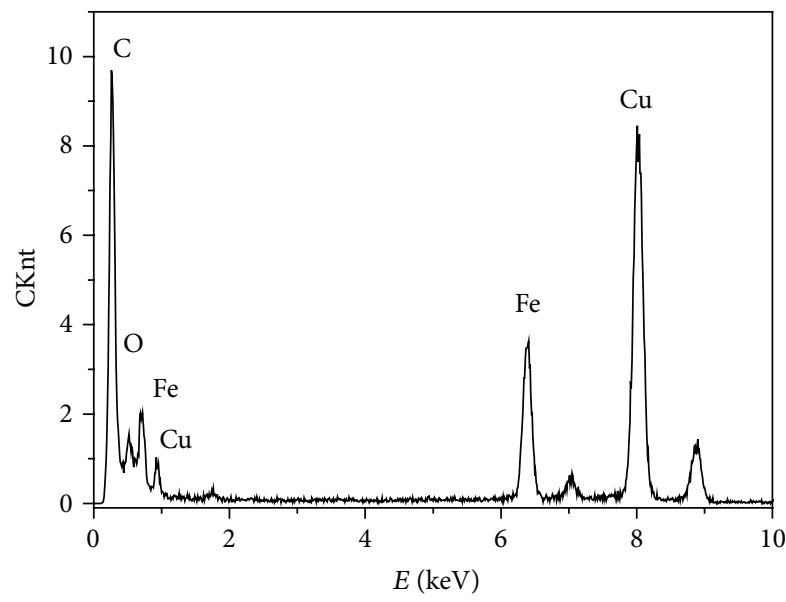

(a)

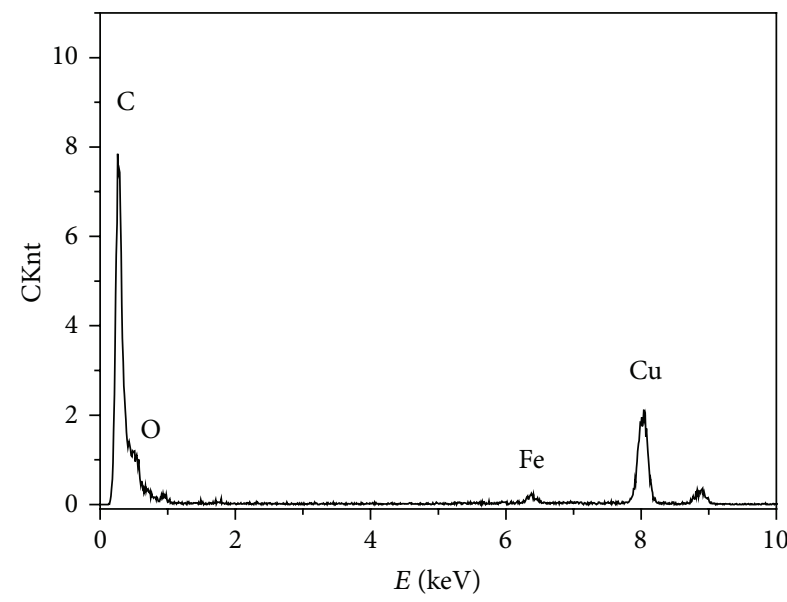

(b)

FIGURE 3: Energy dispersive X-ray spectroscopy (EDS) analysis of magnetic carbon nanocages before (a) and after (b) acid treatment, respectively.

The magnetic carbon nanocages had an average size of about $50 \mathrm{~nm}$ and a shell thickness ranging from 5 to $10 \mathrm{~nm}$. HRTEM image illustrates that the nanocage shell was composed of about 28 well-defined graphitic layers with a spacing of $0.34 \mathrm{~nm}$ (Figures 2(c) and 2(d)), which is consistent with the (002) plane of graphite (Figure 4(a) JCPDS Card number 41-1487). The graphitization degree of the present MCNCs (Figure 2(d)) corresponded well to the results of XRD pattern and Raman spectrum (see below), which are similar to the previous work [43].
The structure of the magnetic carbon nanocages was studied using energy dispersive X-ray spectrometry (EDX). The resulting spectrum is shown in Figure 3. As shown in this figure, the iron particles are present at $0.69,6.41$, and $6.38 \mathrm{keV}$ before and after acid treatment, respectively. The peak at $6.38 \mathrm{keV}$ (Figure 3(b)) can be attributed to the phase of iron particles after acid treatment. Energy dispersive Xray spectroscopy (EDS) analysis (Figure 3(b)) confirmed the TEM result of the few iron nanoparticles residue in MCNCs (Figure 2(b)). 


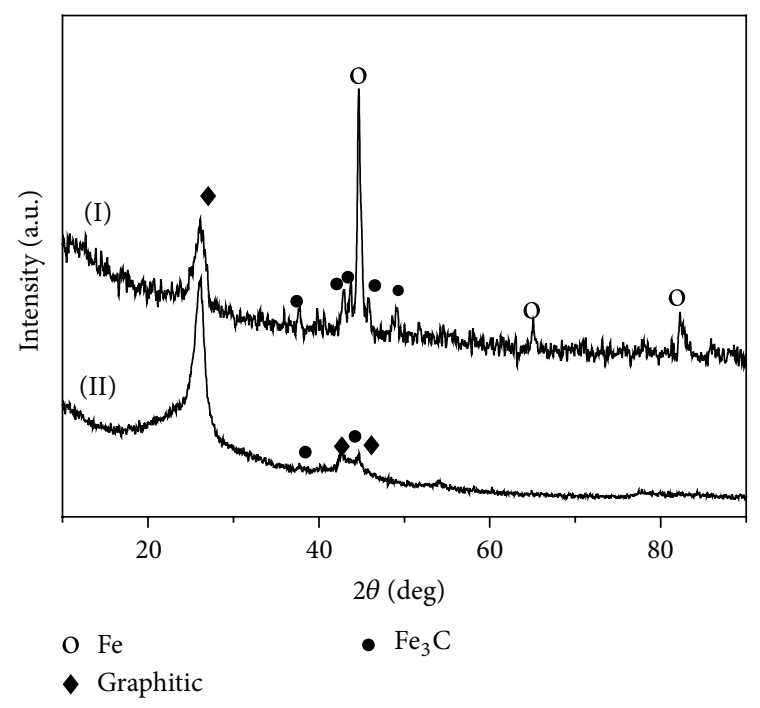

(a)

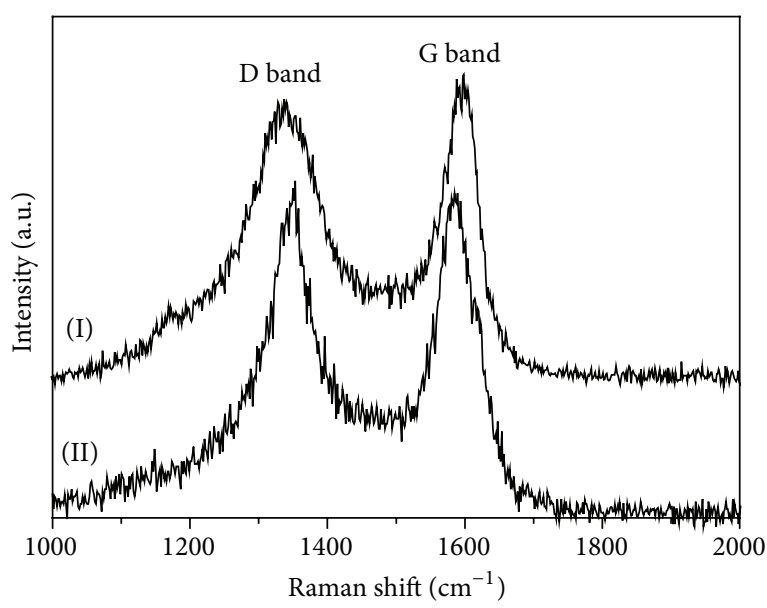

(b)

FIGURE 4: (a) XRD pattern and (b) Raman spectrum of the magnetic carbon nanocages before (I) and after treatment (II).

Figure 4(a) shows the wide-angle X-ray diffraction (XRD) pattern of magnetic carbon nanocages before and after acid treatment; the peaks at around $26.3^{\circ}, 42.2^{\circ}$, and $44.3^{\circ}$ can be indexed as (002), (100), and (101) diffraction planes of the graphite (JCPDS Card number 41-1487), respectively. The weak peaks located at about $37.4^{\circ}, 42.7^{\circ}$, and $43.5^{\circ}$ can be, respectively, indexed to the (121), (211), and (102) reflections of $\mathrm{Fe}_{3} \mathrm{C}$ phases (JCPDS Card number 65-0393), while the other intense peaks at around $44.6^{\circ}, 65.1^{\circ}$, and $82.3^{\circ}$ can be indexed as (110), (200), and (211) diffraction planes of Fe (Figure 4(a), I, JCPDS Card number 65-4899). Compared with the original sample without acid treatment, the peaks of $44.6^{\circ}, 65.1^{\circ}$, and $82.3^{\circ}$ corresponding to Fe phases were no longer observed in the acid-treated sample, suggesting that the Fe was removed, but graphitic layers on the outer surface remained after acid treatment. The reflections of graphitic and $\mathrm{Fe}_{3} \mathrm{C}$ planes were still visible on the XRD pattern of the after acid-treated sample; meanwhile, the peaks of $26.3^{\circ}$ were intense (Figure 4(a), II). Maybe the iron carbides in graphite layers $\left(\mathrm{Fe}_{3} \mathrm{C} @ \mathrm{C}\right)$ are difficult to be acid-treated.

To further investigate the graphitization state of magnetic carbon nanocages before and after acid treatment, Raman spectroscopy test was carried out to define the vibration of carbon species. As shown in Figure 4(b), the magnetic carbon nanocages exhibited a D band at $1338 \mathrm{~cm}^{-1}$ and a $G$ band at $1586 \mathrm{~cm}^{-1}$; the $\mathrm{D}$ band is attributed to an $\mathrm{A}_{1 \mathrm{~g}}$ vibration mode of carbon atoms with dangling bonds in plane terminations of disordered graphite [44]. The G band corresponds to the $\mathrm{E}_{2 \mathrm{~g}}$ mode of hexagonal graphite and is related to the vibration of $\mathrm{sp}^{2}$ hybridized carbon atoms in the graphite layer. The relative intensity of these two bands $\left(\mathrm{I}_{\mathrm{D}} / \mathrm{I}_{\mathrm{G}}\right)$ can be used to reflect the degree of graphitization, which are calculated to be $1.72,1.10$ for before and after acid treatment, respectively. The greater the ratio of $\mathrm{I}_{\mathrm{D}} / \mathrm{I}_{\mathrm{G}}$ is, the higher the disorder degree for graphite is. The relatively lower $I_{D} / I_{G}$ value (1.10) combined with a broad D band confirms that the after acid-treated magnetic carbon nanocages had better graphitization degree [45], which can be confirmed by its XRD pattern (Figure 4(a), II).

Nitrogen sorption isotherms were recorded to investigate the before and after acid treatment of the samples. Figure 5 gives the $\mathrm{N}_{2}$ adsorption-desorption isotherm and corresponding pore size distribution curve of MCNCs. The isotherms (Figure 5(a)) exhibit a clear $\mathrm{H}_{1}$-type hysteresis loop at a relative pressure of $0.5-0.7$, indicating mesoporous characteristics with a capillary condensation phenomenon. The pore size distribution obtained from an analysis of desorption branch of the isotherms is shown in Figure 5(b). It can be seen that the MCNCs sample has bimodal pore size distributions centered at about 4.1, 30-65 nm and 0.57, $3.1,30-65 \mathrm{~nm}$ of magnetic carbon nanocages before and after acid treatment, respectively. These sizes are close to the diameters of the nanocages observed by TEM (Figure 2). Specifically, the pores size $0.57 \mathrm{~nm}$ may be produced by the acid treatment for removal of the metallic iron nanoparticles. These magnetic carbon nanocages generally have high BET surface areas $\left(307.9,420.5 \mathrm{~m}^{2} \cdot \mathrm{g}^{-1}\right)$ and large pore volumes $\left(0.69,0.71 \mathrm{~cm}^{3} \cdot \mathrm{g}^{-1}\right)$; both are increasing with the removal of iron nanoparticles by acid treatment.

The magnetic properties of the synthesized carbon nanocages particles $\left(\mathrm{Fe}_{3} \mathrm{C}\right)$ were characterized by magnetic hysteresis loops with varying magnetic field at room temperature and the obtained magnetization hysteresis loop is shown in Figure 6(a). The hysteresis loop of the carbon nanocages particles $\left(\mathrm{Fe}_{3} \mathrm{C}\right)$ measured at $300 \mathrm{~K}$ shows typical soft magnetic behavior of the composite with a high saturation magnetization up to $19.1 \mathrm{emu} \cdot \mathrm{g}^{-1}$, low coercivity of 136.4 Oe, and low remanence of $2.5 \mathrm{emu} \cdot \mathrm{g}^{-1}$. Figure 6(a) (inset) shows the thermogravimetric analysis (TGA) of the magnetic carbon nanocages materials under the air atmosphere. From 


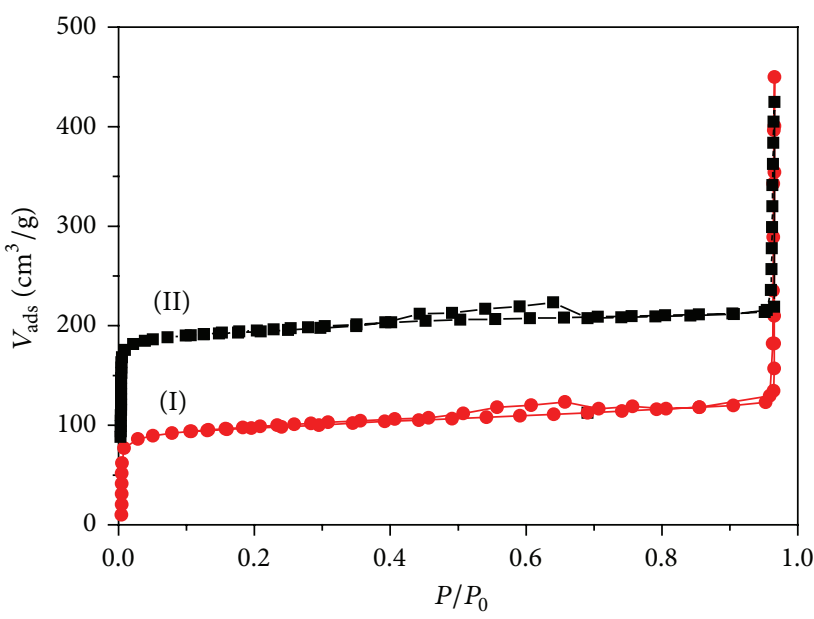

(a)

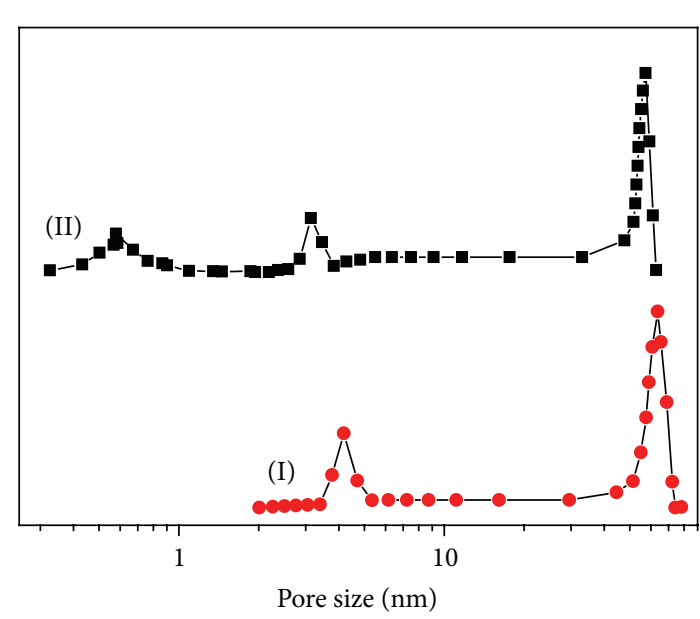

(b)

FIGURE 5: (a) $\mathrm{N}_{2}$ adsorption-desorption isotherms and (b) pore size distribution curves of the magnetic carbon nanocages before (I) and after acid treatment (II).

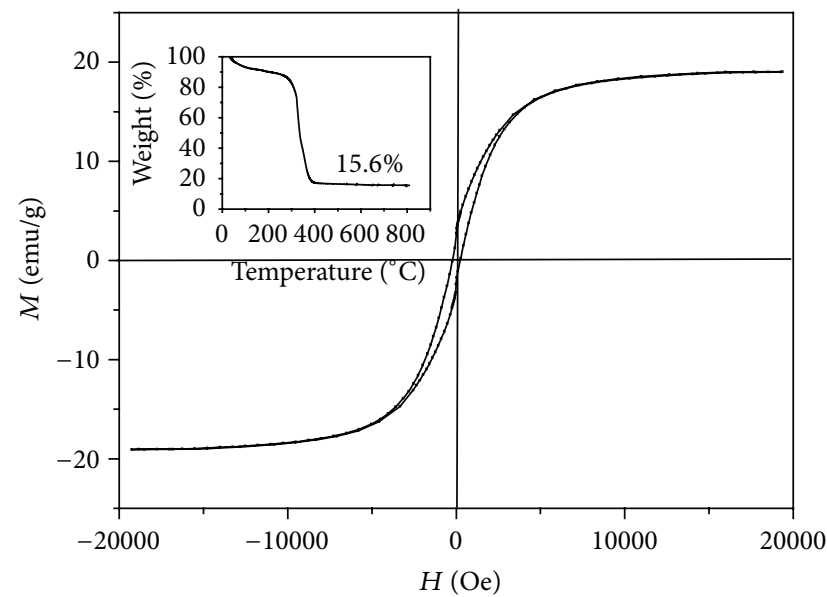

(a)

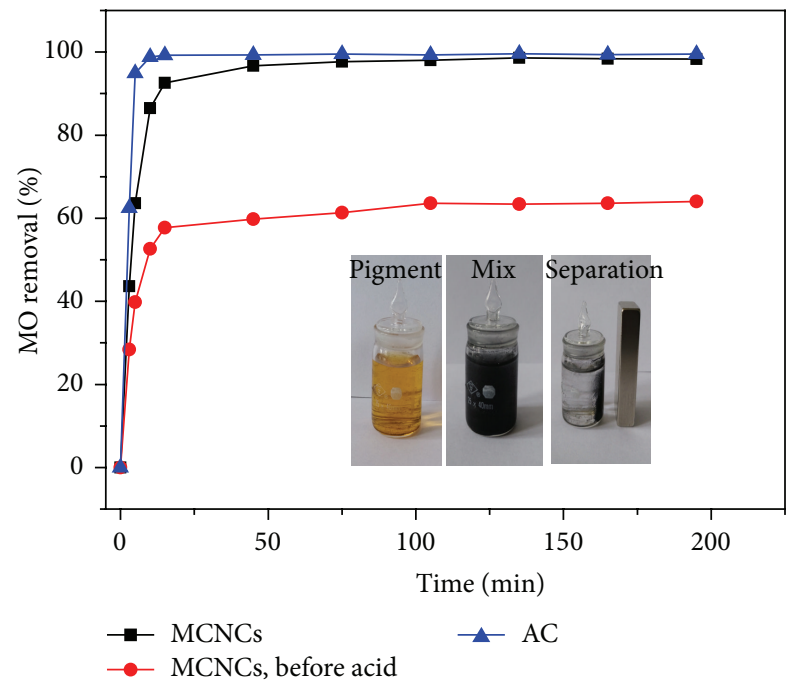

(b)

Figure 6: (a) Magnetization curves of the MCNCs and (b) the adsorption kinetics of MO onto the representative AC and MCNCs. The inset in (a) is the TGA curve of MCNCs. The inset in (b) is a photograph of dispersed MCNCs in MO solution after being attracted by an outer magnet.

TGA results we obtained the iron oxide content which is $15.6 \%$ in MCNCs. Based on calculation, the $\mathrm{Fe}_{3} \mathrm{C}$ content is $17.5 \%$ in MCNCs.

Pollutants, especially textile dyestuffs, have caused overwhelming environmental pollution worldwide, which have triggered many studies' attention. Recent studies showed that magnetic materials with high surface area have a wide potential application for the removal various pollutants in wastewater, notably for textile dyestuffs [46-48]. In order to evaluate the adsorption behavior of magnetic carbon nanocages, we investigate their capacities by adsorbing MO dye from its aqueous solutions. Figure 6(b) presents the adsorption kinetics of $\mathrm{MO}$ dye onto the representative magnetic carbon nanocages with a given initial MO dye concentration of $50 \mathrm{mg} / \mathrm{L}$. MCNCs have obviously better removal ability than that of the MCNs before acid, with adsorption rates of $43.6 \%, 63.6 \%$ and $28.4 \%, 39.8 \%$ in the first 3, $5 \mathrm{~min}$, respectively. Then the dye was rapidly adsorbed in $15 \mathrm{~min}$ (92.6\%), and subsequently the adsorption rate decreased gradually and reached equilibrium in about $60 \mathrm{~min}$ (97.7\%). However, compared with commercial activated carbon, MCNCs adsorption rate is not fast enough, which the adsorption rates of $98.8 \%$ in $10 \mathrm{~min}$.

Magnetic carbon nanocages (MCNCs) exhibit a higher adsorption capacity, which may be attributed to the triple synergy effect based on the special hierarchical pore and 
nanocages structure of MCNCs. Firstly, dye molecules are easily adsorbed or condensed on the exterior surface of MCNCs. Secondly, When the adsorption of the exterior surface reached saturation, the dye molecules extended into the microporous and were adsorbed by the interior surface of MCNCs. Furthermore, it is conjectured that a certain amount of dye molecules may be adsorbed partially or fully filled in the hollow core of MCNCs.

As displayed in Figure 6(b) (inset), the magnetic carbon nanocages can be well-dispersed in MO solution; there was a change from black to colorless within few minutes by placing a conventional laboratory magnet near the glass bottle. That is to say, the black particles of magnetic carbon nanocages were attracted towards the magnet, and the clear solution could be easily decanted off or removed by pipette. It is proved that magnetic carbon nanocages possessed magnetism and could be potentially used as a magnetic adsorbent to remove organic dyes in liquid phase.

\section{Conclusion}

In conclusion, we demonstrate a simple route for the synthesis of magnetic carbon nanocages via a direct carbonization process by using biorenewable lignin as a carbon source. XRD, $\mathrm{N}_{2}$ adsorption-desorption, EDS, VSM, TEM, and Raman results all consistently reveal that the synthesized magnetic carbon nanocages have a multiform pore structure, relative high specific surface area, and relative large pore volume, which are beneficial for the dye molecules diffusion/transfer in the absorbents and thereby result in the improvement of adsorption and separation properties. In addition, the experimental results indicate that the magnetic carbon nanocages exhibited a high capacity for methyl orange adsorption; the excellent adsorption performance of MCNCs can be attributed to its nanocage structure and hierarchical porosity.

\section{Conflict of Interests}

The authors declare that there is no conflict of interests regarding the publication of this paper.

\section{Authors' Contribution}

Hengfei Qin and Yongkui Huang equally contributed to this paper.

\section{Acknowledgments}

This work was carried out with financial supports from National Natural Science Foundation of China (Grant no. 61171008 and no. 21103024). This research was also supported by Yancheng Huanbo Energy Technology Co., Ltd., on field and fund. The authors would like to thank Qinming Liu Professor of Nanjing Forestry University for providing lignin and Chaochuang Yin, Yangling $\mathrm{Xu}$ for experimental technique support.

\section{References}

[1] X. X. Wang, Z. H. Tan, M. Zeng, and J. N. Wang, "Carbon nanocages: a new support material for Pt catalyst with remarkably high durability," Scientific Reports, vol. 4, pp. 4437-4443, 2014.

[2] Y. Yang, Z. J. Wei, C. Y. Wang, and Z. Tong, "Lignin-based Pickering HIPEs for macroporous foams and their enhanced adsorption of copper(II) ions," Chemical Communications, vol. 49, no. 64, pp. 7144-7146, 2013.

[3] W. S. Liu, R. Zhou, D. Zhou et al., "Lignin-assisted direct exfoliation of graphite to graphene in aqueous media and its application in polymer composites," Carbon, vol. 83, pp. 188197, 2015.

[4] M. Y. Chen, Y. B. Huang, H. Pang, X. X. Liu, and Y. Fu, "Hydrodeoxygenation of lignin-derived phenols into alkanes over carbon nanotube supported $\mathrm{Ru}$ catalysts in biphasic systems," Green Chemistry, vol. 17, no. 3, pp. 1710-1717, 2015.

[5] X. Xu, J. Zhou, L. Jiang, G. Lubineau, S. A. Payne, and D. Gutschmidt, "Lignin-based carbon fibers: carbon nanotube decoration and superior thermal stability," Carbon, vol. 80, no. 1, pp. 91-102, 2014.

[6] J. Hayashi, K. Muroyama, V. G. Gomes, and A. P. Watkinson, "Fractal dimensions of activated carbons prepared from lignin by chemical activation," Carbon, vol. 40, no. 4, pp. 630-632, 2002.

[7] S. Chen, J. Y. Bi, Y. Zhao et al., "Nitrogen-doped carbon nanocages as efficient metal-free electrocatalysts for oxygen reduction reaction," Advanced Materials, vol. 24, no. 41, pp. 5593-5597, 2012.

[8] A. V. Erokhin, E. S. Lokteva, A. Y. Yermakov et al., "Phenylacetylene hydrogenation on Fe@C and Ni@C core-shell nanoparticles: about intrinsic activity of graphene-like carbon layer in $\mathrm{H}_{2}$ activation," Carbon, vol. 74, pp. 291-301, 2014.

[9] V. R. Galakhov, A. S. Shkvarin, A. S. Semenova et al., "Characterization of carbon-encapsulated nickel and iron nanoparticles by means of X-ray absorption and photoelectron spectroscopy," Journal of Physical Chemistry C, vol. 114, no. 51, pp. 22413-22416, 2010.

[10] X. Li, F. Gai, B. Guan, Y. Zhang, Y. Liu, and Q. Huo, "Fe@C coreshell and Fe@C yolk-shell particles for effective removal of 4chlorophenol," Journal of Materials Chemistry A, vol. 3, no. 7, pp. 3988-3994, 2015.

[11] C. Xue, Q. Zhang, J. Li et al., "High photocatalytic activity of $\mathrm{Fe}_{3} \mathrm{O}_{4}-\mathrm{SiO}_{2}-\mathrm{TiO}_{2}$ functional particles with core-shell structure," Journal of Nanomaterials, vol. 2013, Article ID 762423, 8 pages, 2013.

[12] G. D. Li, H. X. Yu, L. Q. Xu et al., "General synthesis of carbon nanocages and their adsorption of toxic compounds from cigarette smoke," Nanoscale, vol. 3, no. 8, pp. 3251-3257, 2011.

[13] D. Yuan, J. Chen, J. Zeng, and S. Tan, "Preparation of monodisperse carbon nanospheres for electrochemical capacitors," Electrochemistry Communications, vol. 10, no. 7, pp. 1067-1070, 2008.

[14] R. Feng, L. Wang, Z. Lyu et al., "Carbon nanocages supported $\mathrm{LiFePO} 4$ nanoparticles as high-performance cathode for lithium ion batteries," Acta Chimica Sinica, vol. 72, no. 6, pp. 653-657, 2014.

[15] G. D. Li, L. Q. Xu, Q. Hao, M. Wang, and Y. T. Qian, "Synthesis, characterization and application of carbon nanocages as anode materials for high-performance lithium-ion batteries," RSC Advances, vol. 2, no. 1, pp. 284-291, 2012. 
[16] K. X. Wang, Z. L. Li, Y. G. Wang et al., "Carbon nanocages with nanographene shell for high-rate lithium ion batteries," Journal of Materials Chemistry, vol. 20, no. 43, pp. 9748-9753, 2010.

[17] S. B. Yang, X. L. Feng, L. J. Zhi, Q. A. Cao, J. Maier, and K. Müllen, "Nanographene-constructed hollow carbon spheres and their favorable electroactivity with respect to lithium storage," Advanced Materials, vol. 22, no. 7, pp. 838-845, 2010.

[18] X. Y. Zheng, W. Lv, Y.-B. He et al., "3D hollow Sn@carbongraphene hybrid material as promising anode for lithiumion batteries," Journal of Nanomaterials, vol. 2014, Article ID 974285, 6 pages, 2014.

[19] X. Ji, X. Huang, Q. Zhao, A. Wang, and X. Liu, "Facile synthesis of carbon-coated $\mathrm{Zn} 2 \mathrm{SnO} 4$ nanomaterials as anode materials for lithium-ion batteries," Journal of Nanomaterials, vol. 2014, Article ID 169373, 6 pages, 2014.

[20] J.-Z. Xia, L.-W. Wang, R.-Z. Hu et al., "Enhancement of supercapacitor performance of carbon nanocages by surface functionalization," Chinese Journal of Inorganic Chemistry, vol. 30, no. 9, pp. 2099-2104, 2014.

[21] Y. Tan, C. Xu, G. Chen et al., "Synthesis of ultrathin nitrogendoped graphitic carbon nanocages as advanced electrode materials for supercapacitor," ACS Applied Materials \& Interfaces, vol. 5, no. 6, pp. 2241-2248, 2013.

[22] C. S. Sharma, S. Patil, S. Saurabh, A. Sharma, and R. Venkataraghavan, "Resorcinol-formaldehyde based carbon nanospheres by electrospraying," Bulletin of Materials Science, vol. 32, no. 3, pp. 239-246, 2009.

[23] C. Manaspon, K. Viravaidya-Pasuwat, and N. Pimpha, "Preparation of folate-conjugated pluronic F127/chitosan core-shell nanoparticles encapsulating doxorubicin for breast cancer treatment," Journal of Nanomaterials, vol. 2012, Article ID 593878, 11 pages, 2012.

[24] A. Markwitz, J. Leveneur, P. Gupta, K. Suschke, J. Futter, and M. Rondeau, "Transition metal ion implantation into diamond-like carbon coatings: development of a base material for gas sensing applications," Journal of Nanomaterials, vol. 2015, Article ID 706417, 7 pages, 2015.

[25] C. N. He, F. Tian, S. J. Liu et al., "Characterization and magnetic property of carbon coated metal nanoparticles and hollow carbon onions fabricated by CVD of methane," Materials Letters, vol. 62, no. 21-22, pp. 3697-3699, 2008.

[26] J. Jang and B. Lim, "Selective fabrication of carbon nanocapsules and mesocellular foams by surface-modified colloidal silica templating," Advanced Materials, vol. 14, no. 19, pp. 1390-1393, 2002.

[27] G. D. Li, C. L. Guo, C. H. Sun et al., "A facile approach for the synthesis of uniform hollow carbon nanospheres," The Journal of Physical Chemistry C, vol. 112, no. 6, pp. 1896-1900, 2008.

[28] D. Kawashima, T. Aihara, Y. Kobayashi, T. Kyotani, and A. Tomita, "Preparation of mesoporous carbon from organic polymer/silica nanocomposite," Chemistry of Materials, vol. 12, no. 11, pp. 3397-3401, 2000.

[29] T. Saito, R. H. Brown, M. A. Hunt et al., "Turning renewable resources into value-added polymer: development of ligninbased thermoplastic," Green Chemistry, vol. 14, no. 12, pp. 3295$3303,2012$.

[30] K. Freudenberg, "Biosynthesis and constitution of lignin," Nature, vol. 183, no. 4669, pp. 1152-1155, 1959.

[31] J. M. Pérez, F. Rodríguez, M. V. Alonso, M. Oliet, and J. M. Echeverría, "Characterization of a novolac resin substituting phenol by ammonium lignosulfonate as filler or extender," BioResources, vol. 2, no. 2, pp. 270-283, 2007.
[32] B. Danielson and R. Simonson, "Kraft lignin in phenol formaldehyde resin. Part 1. Partial replacement of phenol by kraft lignin in phenol formaldehyde adhesives for plywood," Journal of Adhesion Science and Technology, vol. 12, no. 9, pp. 923-939, 1998.

[33] Y. P. Zhai, Y. Q. Dou, X. X. Liu, B. Tu, and D. Y. Zhao, “Onepot synthesis of magnetically separable ordered mesoporous carbon," Journal of Materials Chemistry, vol. 19, no. 20, pp. 3292 3300, 2009.

[34] Z. K. Sun, B. Sun, Y. H. Deng, M. H. Qiao, and D. Y. Zhao, "Synthesis of precisely size tunable metallic nanoparticles decorated ordered mesoporous carbon via a general chelateassisted pathway and their remarkable performance in FischerTropsch synthesis," Abstracts of Papers of the American Chemical Society, vol. 243, 2012.

[35] Z. Sun, B. Sun, M. Qiao et al., "A general chelate-assisted co-assembly to metallic nanoparticles-incorporated ordered mesoporous carbon catalysts for Fischer-Tropsch synthesis," Journal of the American Chemical Society, vol. 134, no. 42, pp. 17653-17660, 2012.

[36] M. Zhang and A. A. Ogale, "Carbon fibers from dry-spinning of acetylated softwood kraft lignin," Carbon, vol. 69, pp. 626-629, 2014.

[37] S. X. Hu and Y. L. Hsieh, "Preparation of activated carbon particulates from lignin and its application as electric supercapacitor," in Proceedings of the 247th National Spring Meeting of the American-Chemical-Society (ACS '14), vol. 247 of Abstracts of Papers of the American Chemical Society, Dallas, Tex, USA, March 2014.

[38] P. Estellé, S. Halelfadl, and T. Maré, "Lignin as dispersant for water-based carbon nanotubes nanofluids: impact on viscosity and thermal conductivity," International Communications in Heat and Mass Transfer, vol. 57, pp. 8-12, 2014.

[39] S. Chatterjee, A. Clingenpeel, A. McKenna, O. Rios, and A. Johs, "Synthesis and characterization of lignin-based carbon materials with tunable microstructure," RSC Advances, vol. 4, no. 9, pp. 4743-4753, 2014.

[40] S. J. Dai, S. Y. Yang, and D. Q. Zhou, "The influence of coexisting ions on adsorption-flotation of $\mathrm{Pb}^{2+}$ in water by gordona amarae," Advanced Materials Research, vol. 433-440, pp. 183187, 2012.

[41] Y. Wang, M. Yao, Y. Chen, Y. Zuo, X. Zhang, and L. Cui, "General synthesis of magnetic mesoporous FeNi/graphitic carbon nanocomposites and their application for dye adsorption," Journal of Alloys and Compounds, vol. 627, pp. 7-12, 2015.

[42] J. H. Wang, S. R. Zheng, J. L. Liu, and Z. Y. Xu, “Tannic acid adsorption on amino-functionalized magnetic mesoporous silica," Chemical Engineering Journal, vol. 165, no. 1, pp. 10-16, 2010.

[43] Z. M. Sheng and J. N. Wang, "Thin-walled carbon nanocages: direct growth, characterization, and applications," Advanced Materials, vol. 20, no. 5, pp. 1071-1075, 2008.

[44] M. Ginic-Markovic, J. G. Matisons, R. Cervini, G. P. Simon, and P. M. Fredericks, "Synthesis of new polyaniline/nanotube composites using ultrasonically initiated emulsion polymerization," Chemistry of Materials, vol. 18, no. 26, pp. 6258-6265, 2006.

[45] T.-W. Kim, I.-S. Park, and R. Ryoo, "A synthetic route to ordered mesoporous carbon materials with graphitic pore walls," Angewandte Chemie International Edition, vol. 42, no. 36, pp. 4375-4379, 2003.

[46] A. Banerjee, R. Gokhale, S. Bhatnagar et al., "MOF derived porous carbon- $\mathrm{Fe}_{3} \mathrm{O}_{4}$ nanocomposite as a high performance, 
recyclable environmental superadsorbent," Journal of Materials Chemistry, vol. 22, no. 37, pp. 19694-19699, 2012.

[47] C. Zhang, Z. L. Mo, P. Zhang, C. Feng, and R. B. Guo, "Facile synthesis of porous carbon@ $\mathrm{Fe}_{3} \mathrm{O}_{4}$ composites and their applications in wastewater treatment," Materials Letters, vol. 106, pp. 107-110, 2013.

[48] N. Yang, S. M. Zhu, D. Zhang, and S. Xu, "Synthesis and properties of magnetic $\mathrm{Fe}_{3} \mathrm{O}_{4}$-activated carbon nanocomposite particles for dye removal," Materials Letters, vol. 62, no. 4-5, pp. 645-647, 2008. 

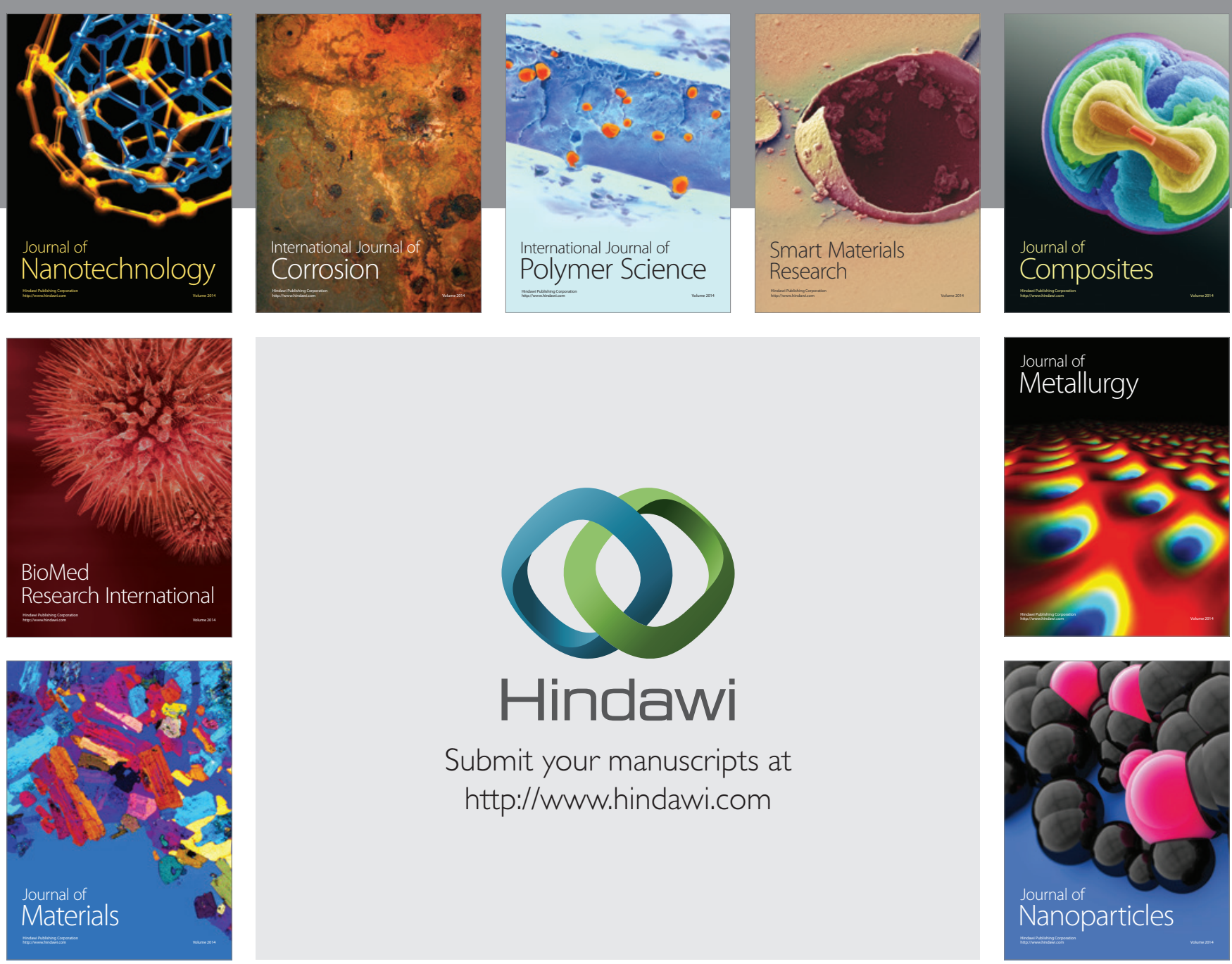

Submit your manuscripts at http://www.hindawi.com
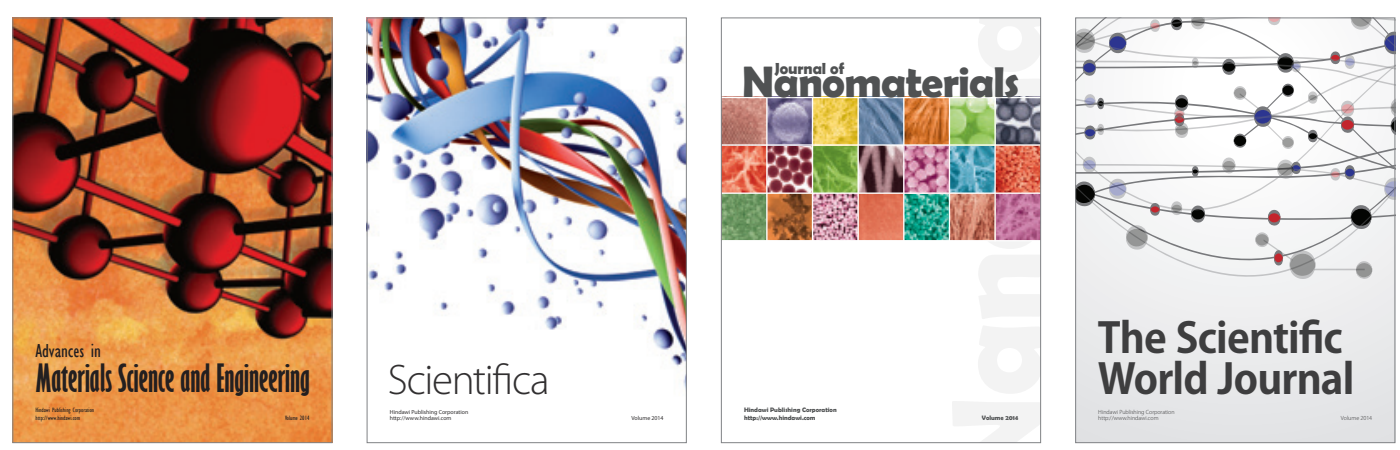

\section{The Scientific World Journal}
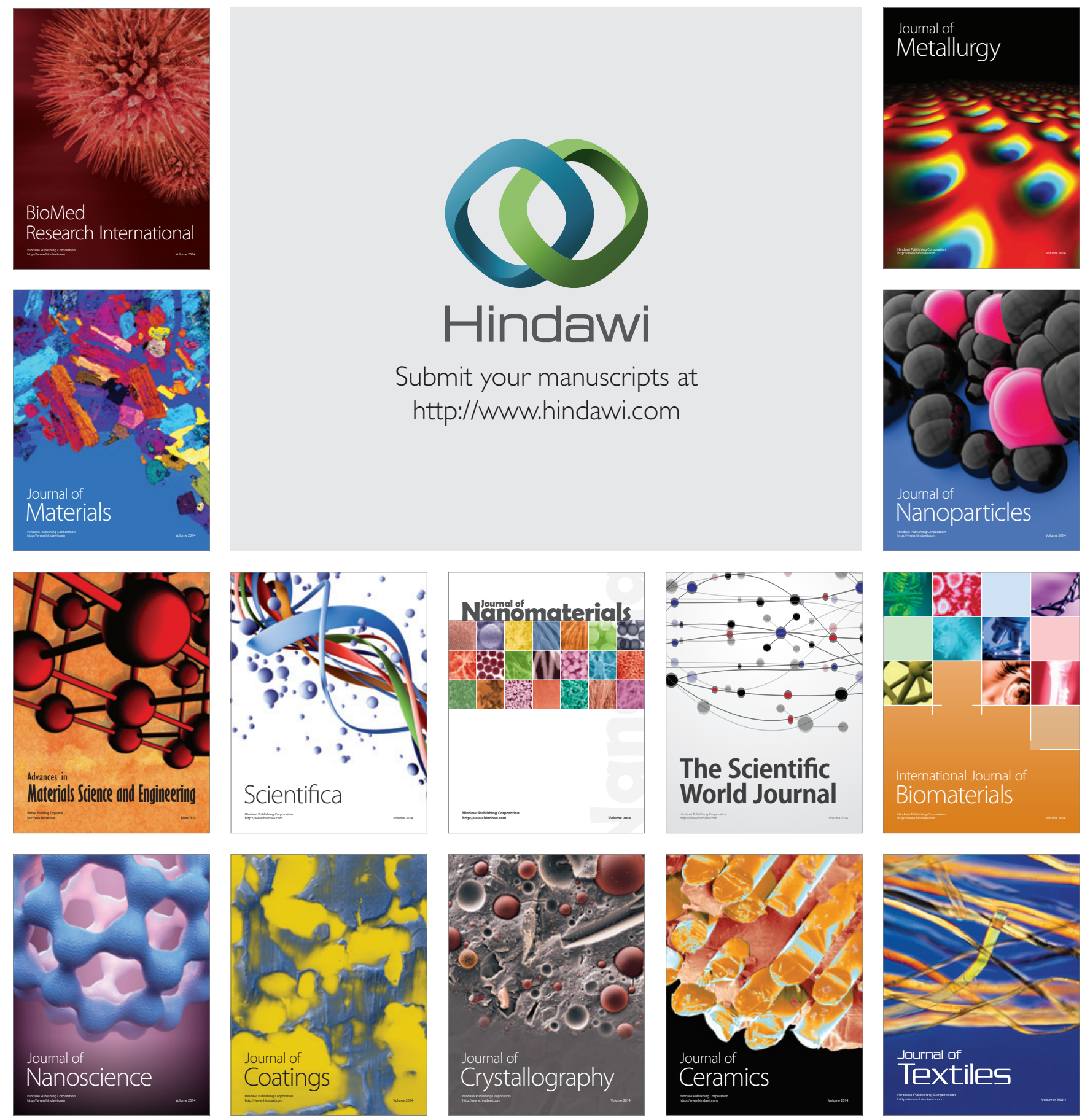